home-collection kit included a self-collected vaginal swab (women), UriSWAB (Copan) for urine collection (heterosexual men), and UriSWAB plus rectal swab (MSM). The primary outcome was the proportion retested at 1-4 months after chlamydia diagnosis, and the secondary outcome was the proportion with repeat positive results at the 1-4 month retest. Any testing outside the study sites was collected and included in the outcomes. An intention to treat analysis was conducted.

Results Overall 61\% (183/300) of home group participants retested within 1-4 months of chlamydia diagnosis compared with $39 \%$ $(118 / 300)$ in the clinic group $(p<0.001)$. According to risk group, the differences were: $62 \%$ vs $45 \%$ (MSM); $65 \%$ vs $38 \%$ (women); and $55 \%$ vs $34 \%$ (heterosexual men); all $p<0.05$. Overall the proportion with a repeat positive result at the 1-4 month re-test was 16\% (95\% CI: 11-23) (30/183) in the home group compared with 10\% (95\% CI: $5-17)(12 / 118)$ in the clinic group: $26 \%(95 \%$ CI: $16-39)$ vs $11 \%(95 \%$ CI: 4-24) MSM; $12 \%$ (95\% CI: 5-22) vs 5\% (95\% CI: $1-18)$ women; and $11 \%$ (95\% CI: $4-22)$ vs $15 \%$ (95\% CI: $5-31)$ heterosexual men.

Conclusion SMS reminders combined with home-based collection was a very effective strategy to increase chlamydia retesting in all three risk groups, and also detected additional repeat infections in MSM. The acceptability to patients and health care provider costs are currently being evaluated.

\subsection{3 - Risk behaviours and preventive interventions}

\subsection{SEXUAL RISK TRAJECTORIES AMONG MSM IN THE UNITED STATES: IMPLICATIONS FOR PREP DELIVERY}

doi:10.1136/sextrans-2013-051184.0216

${ }^{1} \mathrm{H}$ A Pines, ${ }^{1} \mathrm{P} M \mathrm{M}$ Gorbach, ${ }^{2} \mathrm{R}$ E Weiss, ${ }^{3} \mathrm{~S}$ Shoptaw, ${ }^{4,5} \mathrm{D} \mathrm{G}$ Ostrow, ${ }^{6} \mathrm{R}$ D Stall, ${ }^{7} \mathrm{M}$ Plankey. 'Department of Epidemiology, University of California, Los Angeles Fielding School of Public Health, Los Angeles, CA, United States; ${ }^{2}$ Department of Biostatistics, University of California, Los Angeles Fielding School of Public Health, Los Angeles, CA, United States; ${ }^{3}$ Department of Family Medicine, Department of Psychiatry and Biobehavioral Sciences, University of California, Los Angeles, Los Angeles, CA, United States; ${ }^{4}$ David Ostrow \& Associates, LLC, Chicago, IL, United States; ${ }^{5}$ The Chicago MACS, Northwestern University, Evanston, IL, United States; ${ }^{6}$ Department of Behavioral and Community Health Sciences, Graduate School of Public Health, University of Pittsburgh, Pittsburgh, PA, United States; ' Department of Medicine, Georgetown University Medical Center, Washington, DC, United States

Background CDC guidelines state that men who have sex with men (MSM) at ongoing high risk of HIV infection should be targeted for pre-exposure prophylaxis (PrEP). Longitudinal data can inform the implementation of these guidelines.

Methods HIV-seronegative MSM enrolled in the Multicenter AIDS Cohort Study at 4 U.S. sites completed ACASIs at semiannual visits. Behaviors since the last visit from 10/1/20039/30/2011 were used to assign participants sexual risk behaviour (SRB) scores ranking their risk level at each visit: (0) no insertive and/or receptive anal intercourse (IAI/RAI), (1) no unprotected IAI and/or RAI (UIAI/URAI), (2) only UIAI, (3) URAI with 1 HIV-negative partner, (4) condom-seropositioning, (5) condom-serosorting, and (6) no seroadaptive behaviours. Group-based trajectory modelling was used to examine SRB scores ( $<4$ vs. $\geq 4)$ and identify groups with distinct patterns of sexual risk.

Results The sample $(\mathrm{N}=430)$ was $38.4 \%$ White, $42.3 \%$ Black, $14.9 \%$ Hispanic and had a median age of 39.1 years $(\mathrm{IOR}=31.3-$ 44.3). Three trajectory groups were identified: no risk $(\mathrm{N}=286$; $66.5 \%)$, low risk $(\mathrm{N}=89 ; 20.7 \%)$, and high risk $(\mathrm{N}=55 ; 12.8 \%)$. Compared to the no risk group, high risk group membership was negatively associated with older age (adjusted odds ratio [AOR] for 5 -year age difference $=0.68,95 \%$ CI: $0.56-0.84$ ) and positively associated with being White (AOR $=2.12,95 \% \mathrm{CI}$ : 0.97-4.62), earning an income $\geq \$ 20,000$ ( $\mathrm{AOR}=4.96,95 \% \mathrm{CI}: 2.10-11.71$ ), depression $(\mathrm{CESD} \geq 16)(\mathrm{AOR}=2.06,95 \% \mathrm{CI}: 0.98-4.31)$, and stimulant use $(\mathrm{AOR}=2.37,95 \% \mathrm{CI}: 1.18-4.78)$ at the index visit. Adjusted group membership probabilities for a 30 year-old, White male reporting an income $\geq \$ 20,000$, depression, and stimulant use at the index visit were 0.15 (no risk), 0.39 (low risk), and 0.46 (high risk).

Conclusion Findings suggest MSM following high risk trajectories could be identified by the socio-demographic and behavioural factors described above, thus enabling PrEP programmes to target those at ongoing high risk of HIV infection.

\subsection{CORRELATES OF INCONSISTENT CONDOM USE DURING ANAL SEX WITH FEMALE SEX WORKERS (FSWS) AMONG MALE CLIENTS: SURVEY FINDINGS FROM THREE HIGH PREVALENCE STATES OF INDIA}

doi:10.1136/sextrans-2013-051184.0217

'S Ramanathan, 'L Ramakrishnan, 'P Goswami, 'D Yadav, 'B George, 'S Sen, ${ }^{2} \mathrm{~V}$ Chakrapani, ${ }^{3} \mathrm{R}$ Paranjape, ${ }^{4} \mathrm{~T}$ Subramanian, ${ }^{5} \mathrm{H}$ Rachakulla. ${ }^{1} \mathrm{FHI} 360$, New Delhi, India; ${ }^{2}$ Centre for Sexuality and Health Research and Policy (C-SHaRP), Chennai, India; ${ }^{3}$ National AIDS Research Institute (NARI), Pune, India; ${ }^{N}$ National Institute of Epidemiology (NIE), Chennai, India; ${ }^{5}$ National Institute of Nutrition (NIN), Hyderabad, India

Background Recent studies from India show that self-reported anal sex increased from $3 \%$ to $22 \%$ among female sex workers. However, comparable data from male clients' of FSWs are lacking. Using data from a bio-behavioural survey (2009-2010), we examined correlates of male clients' self-reported inconsistent condom use during anal sex with FSWs in Andhra Pradesh, Maharashtra and Tamil Nadu.

Methods Using a two-stage time-location cluster sampling, we recruited 4,803 men aged between 18 and 60 years who purchased sex from FSWs in the past month. After obtaining informed consent, respondents were interviewed and tested for HIV and STIs (Syphilis, Gonorrhea and Chlamydia). Logistic regression analysis was used to identify factors associated with inconsistent condom use during anal sex (in the past six months) with FSWs controlling for socio demographics and other contextual characteristics.

Results Overall, $12.4 \%$ clients reported anal sex in the past 6 months and nearly half $(48.4 \%)$ used condoms inconsistently. Majority of these inconsistent users solicited FSWs from public places (77\%), consumed alcohol (50\%), had unprotected vaginal sex (99\%) and also reported anal sex with other men (19\%). Factors associated with increased odds for inconsistent condom use were being aged above 25 years (AOR:3.38, $\mathrm{p}=0.012$ ), occupation as manual labourer (AOR:2.05, $p=0.029)$ and perceiving to be at risk of HIV (AOR:10.2, $p=0.000$ ) Those literate (AOR:0.40, $p=0.033$ ) and currently married (AOR:0.41, $\mathrm{p}=0.056$ ) were at decreased odds and being STI/HIV positive was not significantly associated with inconsistent condom use.

Conclusion Results suggest that a relatively high proportion of clients were not using condoms consistently during anal and vaginal sex with FSWs which implies a greater risk of acquiring HIV and its further transmission to their male and female sexual partners (including spouses). Given the multidirectional risk, safer sex messages addressing heterosexual anal sex needs to be incorporated into HIV prevention interventions.

\subsection{PREDICTORS OF SELF-ASSESSED RISK OF CHLAMYDIA TRACHOMATIS INFECTION AMONG ADOLESCENTS IN NORWAY}

doi:10.1136/sextrans-2013-051184.0218

${ }^{1} \mathrm{~K}$ Gravningen, ${ }^{2} \mathrm{H}$ Schirmer, ${ }^{1} \mathrm{~A}$ Furberg, ${ }^{1} \mathrm{G}$ S Simonsen, ${ }^{3} \mathrm{~T}$ Wilsgaard. ${ }^{1}$ University Hospital of North Norway, Tromsø, Norway; ${ }^{2}$ Institute of Clinical Medicine, University of Tromsø, Tromsø, Norway; ${ }^{3}$ Department of Community Medicine, University of Tromsø, Tromsø, Norway 
Background Adolescents commonly underestimate their own risk of chlamydia infections. We aimed to examine the association between chlamydia prevalence and perceived risk of infection, and the predictors of perceived risk, among 15-20 year old adolescents in Norway.

Methods This population based cross-sectional study among students in 5 high schools included a web-questionnaire and Chlamydia trachomatis PCR in first-void urine (participation rate 85\%, girls 800/ boys 818 , mean age 17.2 years). Perceived infection risk was assessed using a 5 -point scale: $1=$ none, $2=$ low, $3=$ medium, $4=$ high, $5=$ very high. Multivariable logistic regression analysis was applied using stepwise variable selection with $5 \%$ significance level and binary outcome: 'high' (3-5) versus 'low' (1-2) risk.

Results Chlamydia prevalence according to risk level was: none $3.6 \%$, low $4.4 \%$, medium $8.2 \%$, and high/very high $16.4 \%$. 28 of the 58 infected participants perceived their risk to be none/low. In the multivariable model, following factors were associated with high perceived risk: $\geq 2$ sexual partners past 6 months (odds ratio (OR) 3.6), number of lifetime partners 1-2 (reference), 3-5 (OR 2.7), or $\geq$ 6 (OR 3.7), previous treatment (OR 2.2), clinical symptoms (OR $1.9)$, no steady relationship (OR 1.8), first intercourse without condom (OR 1.5), and younger age (OR 0.78 per year). Significant interaction was present between gender and substance use $(p=0.004)$. Higher levels of substance use increased self-assessed risk only among boys. Among participants with low use, boys assessed risk higher than girls (OR 2.7). Among medium use participants, boys assessed risk lower than girls (OR 0.6). Among 665 participants perceiving none/low risk, both the 28 persons infected and a significant proportion of the non-infected scored on multiple chlamydia risk factors.

Conclusions Chlamydia prevalence increased with increasing perceived risk level. Mostly well-known chlamydia risk factors were significant in modelling risk perceptions. Adolescents need knowledge to more accurately assess their susceptibility to chlamydia infections.

\subsection{UNDERSTANDING DOMESTIC VIOLENCE AS A PREDICTOR OF ADVERSE HEALTH OUTCOMES AND SEXUAL RISK- TAKING AMONG MEN WHO HAVE SEX WITH MEN (MSM): A SYSTEMATIC REVIEW AND META-ANALYSIS}

doi:10.1136/sextrans-2013-051184.0219

A Buller, L Bacchus, K Devries. London School of Hygiene and Tropical Medicine, London, UK

Between $15.4 \%$ and $51 \%$ of men who have sex with men (MSM) have experienced domestic violence (DV). The adverse health consequences of DV are well documented for women victims, but little is known about the association between DV and health outcomes for men who have sex with men. We aimed to establish if MSM exposed to DV had higher odds of experiencing adverse health outcomes or engaging in risk behaviours. In this systematic review and metaanalysis, we searched 13 electronic databases to identify primary research studies reporting the risk of adverse health outcomes or risk behaviours for MSM experiencing or perpetrating DV. Of 4,653 identified, 17 were included in the review, of which 15 were cross-sectional and 2 cohort. The 17 studies described 16 datasets with 12,778 participants, reporting 82 estimates. All studies were included in the meta-analysis. We found that exposure to DV increased the odds of substance use (1.68, 95\% CI 1.44-1.92), being HIV positive (1.4, 95\% CI 1.19-1.62) and depression (1.6, 95\% CI 1.28-1.92). Pooled crude odds ratios of the risk of unprotected anal sex in MSM exposed to DV were $(1 \cdot 49,95 \%$ CI $0 \cdot 67-2 \cdot 30)$ with high level of heterogeneity among estimates. We conclude that DV has adverse health consequences for MSM. Exposure to DV is a risk factor for substance use, depression and HIV status. The evidence for the association between
DV and sexual risk behaviour such as unprotected anal sex is less robust. There is a lack of cohort studies which limits the possibility to establish the direction of these associations. Studies are absent from Europe and low and middle income countries.

\section{5 "CIRCUMCISION MAKES THEM HALF MEN": CONCERNS ABOUT LOSS OF MASCULINITY MAY DETER ACCEPTANCE OF SAFE MEDICAL MALE CIRCUMCISION}

doi:10.1136/sextrans-2013-051184.0220

'P Lilleston, ${ }^{2} \mathrm{~A}$ V Marcell, ${ }^{3} \mathrm{~N}$ Nakyanjo, ${ }^{1} \mathrm{~L}$ Leonard, ${ }^{4} \mathrm{M} \mathrm{J}$ Wawer. ${ }^{1}$ Department of Health, Behavior, and Society, Johns Hopkins Bloomberg School of Public Health, Baltimore, MD, United States; ${ }^{2}$ Department of Population, Family and Reproductive Health, Johns Hopkins Bloomberg School of Public Health, Baltimore, MD, United States; ${ }^{3}$ Rakai Health Sciences Program, Entebbe, Uganda; ${ }^{4}$ Department of Epidemiology, Johns Hopkins Bloomberg School of Public Health, Baltimore, MD, United States

Background Despite access to safe medical male circumcision (MMC) and the proven effectiveness of the procedure in reducing acquisition of HIV and other STIs, uptake remains relatively low in Rakai District, Uganda. Gender may play an important role in use of HIV prevention services, yet few studies have examined linkages between beliefs about what it means to be a man and acceptance of MMC.

Methods We explored this relationship in focus group discussions ( $n=34$ groups) with men and women in Rakai. Focus groups were conducted from May through July, 2012 with adolescent and adult males with a range of HIV risk and reproductive health service use profiles, and with adolescent and adult females. Data were analysed using Atlas-ti and an inductive approach.

Results Participants' beliefs about manhood were grounded in the concepts of responsibility (signified through provision of economic and social stability in the family), independence, sexuality, fertility, and religiosity. While some participants described MMC as leading to more pleasurable sex and better hygiene, other aspects of MMC were perceived as threatening valued aspects of manhood. For instance, the post-surgical healing period required time off work and increased dependence on family support. Males worried about restrictions on sexual activity during the healing period and the perceived side-effects of the procedure, such as reductions in sexual drive. Some Christian participants equated male circumcision with religious conversion or the desecration of the body as God's creation. Women reported that MMC could be an indicator of infidelity in a relationship, inciting conflict between sexual partners.

Conclusion In considering MMC, males weighed the potential benefits of avoiding HIV infection in the future against more immediate threats to their sense of self as men. Understanding how males and females view MMC is a crucial step towards increasing uptake of the procedure and reducing disease transmission.

\subsection{FACTORS THAT INFLUENCE CONTRACEPTIVE CHOICE AND USE AMONG HIV DISCORDANT COUPLES IN KISUMU, KENYA}

doi:10.1136/sextrans-2013-051184.0221

'J B Odoyo, 'B Rono, ' ${ }^{1} \mathrm{~K}$ Owuor, ${ }^{2} \mathrm{C}$ R Cohen, 'E A Bukusi. 'Kenya Medical Research Institute, Nairobi, Kenya; ${ }^{2}$ University of California San Francisco, San Francisco, CA, United States

Background Factors influencing choice and use of contraceptives among HIV discordant couples have important policy and programmatic implications for HIV and pregnancy prevention. We investigated factors among women and men in an HIV serodiscordant couple enrolled in a phase 3 clinical trial of Partners PrEP study. Methods Prospective sub-study enrolled 385 men and women selected from 629 couples. Family planning counselling and contraceptives were offered to participants at each visit. 